\title{
Simulation, analysis and service quality in carrier voice networks
}

\author{
Marius Popescu ${ }^{1}$, Andrei Gabor ${ }^{1}$ and Antoanela Naaji ${ }^{1, a}$ \\ “"Vasile Goldis” Western University of Arad, Engineering and Computer Science Department, 86 Rebreanu Str., Romania
}

\begin{abstract}
Due to existing national regulations in the field of telecommunications, sometimes conflict situation arise between telecommunication operators. In these cases performing simulations and quality analysis are required. The paper describes the ways and means of changing caller ID in the relationship established between two telecommunication operators of different ranks, which have concluded a service agreement. Simulating the mode of interconnection between operators, based on an interface represented by Voice Gateway, and through various settings on a dial plan, one can establish a real operation. Finally, traffic trends are studied over a period of time, analyzing the conflicts started between the two telecommunications operators regarding traffic and quality of services.
\end{abstract}

\section{Introduction}

After 2000, Europe has seen the emergence of operators developing their own telecommunication networks, which have helped improve speed in accessing telecommunication services, quickly attaining the appropriate level of user requirements. During this time, in Romania the national regulatory authority in telecommunications (ANCOM) defined:

- carrier operators and/or service providers as those with "significant power ... of broadband electronic communication services and public telephone services at fixed points", who can directly provide users with Internet access services;

- dominant operators as those with "significant market power in terms of access to their mobile network in order to terminate calls", requiring interconnection with any other carrier operator and/or telecommunication service provider.

As a result, agreements have emerged between carrier operators and/or service providers and dominant operators, stipulating the installation of bi-directional communication streams (e.g. 30 channels, E1 [1]), without establishing details on how to identify and resolve deviations from prerequisites such as exceeding the value of daily traffic (e.g. traffic exceeding 30,000 minutes/month), forced route "maturing" (e.g., provided after 6 months), etc. Services required by service provider operators from the dominant operator were:

- termination of calls originated by customers of the carrier operator and /or service provider to the dominant operator's network;

- termination of calls originated by the dominant operator's network to the geographic numbers allocated to the carrier operator and/or service provider (for example, Romania: $021569 \operatorname{xxxx}$ where $\mathrm{xxxx}=0,1, \ldots .9$ );
- routing calls originated by the dominant operator's network to the carrier operator and/or service provider's exchange for calls with carrier selection;

- termination of calls originated by the dominant operator network to non-geographic numbers allocated to the carrier operator and/or service provider (e.g., green numbers $0800120 \mathrm{xxx}$, prepaid cards $0808810 \mathrm{xxx}$, or value added services $0903030 \mathrm{xxx}$, where $\mathrm{x}=01, \ldots, 9$ ); allocation of short $\mathrm{xxxx}$ numbers, callable from the dominant operator's network, and which are routed to non-geographic numbers of the carrier operator and/or service provider, providing various services to customers depending on the set category.

- line services leased between customers and the company of the carrier operator and/or service provider through the dominant operator's network.

In our paper we present a real situation, in which traffic estimates for services established between the dominant operator and the service provider operator was 400,000 minutes/month in the first 3 months, of which $100 \%$ voice - to - voice calls, which in turn was distributed in a proportion of $70 \%$ over the $08-18$ time slot and 30\% over the 18-08 time slot. Transmission hours were spread evenly from Monday to Sunday, with peak traffic from 10 am to $4 \mathrm{pm}$. The maximum number of simultaneous calls was 70, and the prospects for traffic increase were determined, depending on services, at 2 million minutes per month for the next eight months. We used numerical simulation for the quality analysis because in some cases there is no possibility to make this evaluation directly on equipment. For testing the method, we compared the results with those obtained through experimental evaluation. As conclusion, we demonstrated that the increasing trends of traffic over a short period of time (e.g. 20 days) resulted in the dominant operator taking additional measures to monitor traffic and

${ }^{\mathrm{a}}$ Corresponding author: anaaji@uvvg.ro 
moderate its growth. This fact caused discontent in the carrier operator and/or service provider, the latter mentioning that the traffic of their subscribers is a process that evolves in a probabilistic manner which cannot be controlled, and therefore requiring a new interconnection agreement for national and international call routing to the dominant operator's network.

\section{Hardware and software used by telecommunications operators}

\subsection{Methods and equipment for the interconnection of telecommunication operators}

The physical equipment (hardware) used by the carrier operator and/or service provider to connect with the dominant operator was an Ericsson ANS Translocal R420R2 exchange [2] with Diax A/S NMLCM v5 .43 support/OS/software, using SS7 CCS7v2.2 signalling, which allowed routing national and international calls to the dominant operator's network. Equipment used (in 2004, similar to Alcatel equipment) by the dominant operator had the following specifications: SW releases [3] SW of the platform (according to existing HW) are: Evolium SSP release R272.3.4.2/V9 (E10), Evolium RCP release R909 (EP8) and Evolium M HLR release GB910 (EP9). The gatekeeper, which provides call control for endpoints, more streamlined for incoming calls, is preceded by other equipment. Thus, VoIP calls incoming from interconnection lines of the input port, where transmission is performed by packets, were taken from an IP switch (DJAX-AS) layer 2 or 3 and, if the numbers were specific to the dominant operator, they were then routed to the receiving interface port. In the case of the service operator, the IP switch type (DJAX-AS) was layer 3 (in OSI - Open Systems Interconnection architecture) and therefore it could provide, in addition to the outgoing address, the input port address and not that of the caller. Figure 1 shows the configuration of interconnections in the case of incoming calls in the carrier operator and/or service provider network from other national and international networks.

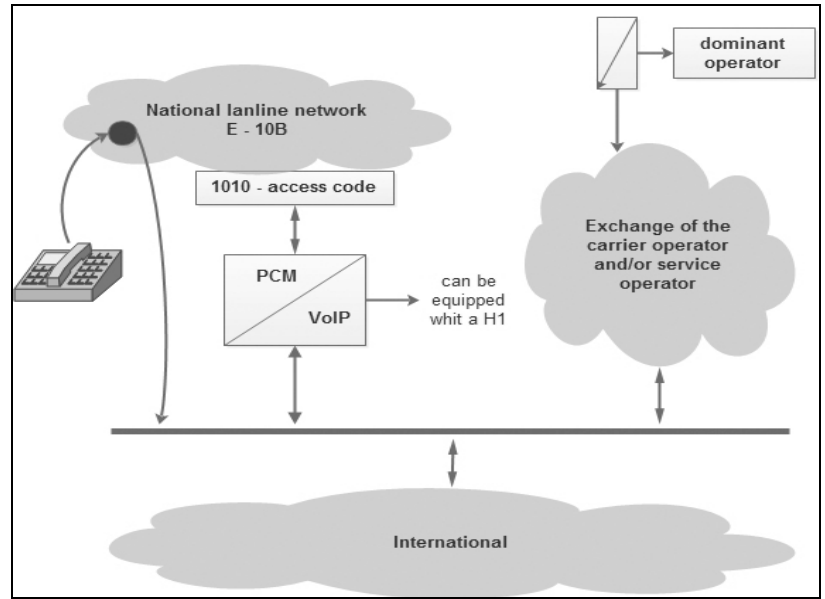

Figure 1. Explanatory scheme on the direct interconnection between the carrier operator and/or service provider network and the landline network (Alcatel) via an adapter (of the same type as for the interconnection with the dominant operator.
The access code in 2004, established under the interconnection agreement of the carrier operator and/or service provider with the national landline network, was changed in 2008 by ANCOM into access code 1010, according to the National Numbering Plan.

The equipment used proved that the carrier operator and/or service provider does not preserve caller ID (e.g., 021-BPQMCDU), introducing its own authorized numbering (021-569xxxx). Or, in order to change caller ID, the operation might be assigned (allocated) to either: a) the layer 3 switch of the VoIP equipment (the carrier operator and/or service provider exchange);

b) or directly in the channel interface - 1 - MUX converter PCM/VoIP used for interconnecting the VoIP exchange with the mobile and landline network (gatekeeper).

In the first case, each incoming channel from platform 1010 is assigned from the outset a specific numbering on the VoIP network of the carrier operator and/or service provider, such as $021569111 \mathrm{x}$ (authorized numbering, the tranche of numbers used being highlighted in bi-annual reports of the carrier operator and/or service provider to ANCOM). In case of the carrier operator and/or service provider declares that the number of its subscribers is 11 , 12 or 15 , is sufficient to be used and allocated as ID to incoming channels on the landline or international network.

In the second case, if a simple converter is used, with no processing capacity, the ID change is performed within the layer 3 switch, the other numbering problems being the same as in the previous case. Connection with the dominant operator on the output of the VoIP - PCM converter is equipped with a 30-channel MUX, and not E1 stream. For calls originating in the dominant operator's network, calls will be connected directly via VoIP network - 4 - bypassing platform 1010 (Figure 2). H.323 (1996) is a suite of protocols that includes both signalling protocols (signalling and controlling point-topoint and multi-point conferences - calling, data bandwidth, etc.), as well as transport protocols. The H.323 standard describes a series of functional components, which can be implemented separately in different equipment, or can be grouped into one multipurpose equipment.

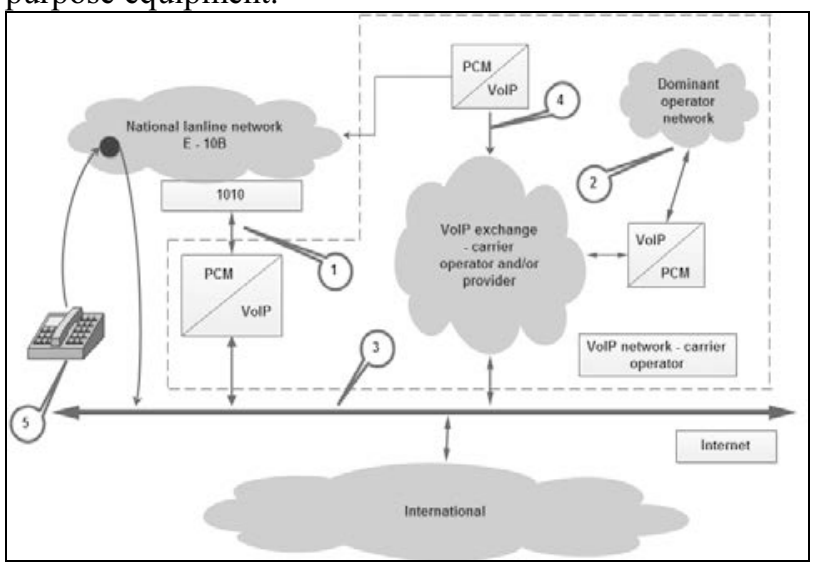

Figure 2. Explanatory scheme on the physical interconnection of the network operator and/or service provider network with the Internet data network. 
For the first 10 months, the numbering plan used by the carrier operator and/or service provider analyzed in this case was limited to 10,000 call numbers (required by the equipment used [4]). Thus, as recommended by ITU$\mathrm{T}$ (International Telecommunication Union), it could not be considered a public telecommunication service operator, but at most a VPN (Virtual Private Network) [5] implemented in the network of another dominant operator or a service provider between networks (ISP Internetwork Service Provider). The carrier operator and/or service provider could allow routing, through its network, national and international calls from other national telecommunication operators to subscribers of the dominant operator with which they concluded an agreement, but only if this behavior was tolerated by the dominant operator.

\subsection{Simulating an interconnection situation}

Since it is not possible to carry out experimental simulations on a model that has its 2004 configuration, mainly for the analysis of an E1 stream as a link between a communication service distributor (Ericsson Diax Ans) and the dominant operator network as transit network (Alcatel 1000 E10 OCB 283 HC [1]), under certain circumstances, different software can be used to simulate the situation described earlier in this paper. Specifically to achieve such goals, a medium that simulates the infrastructure of some operators was used, in this case the connection between the dominant operator and the carrier operator.

Emulator GNS3 [6] and the application Cisco Unified Communication Manager (CUCM) [7] were used for simulation, the latter being an integrated communication system, based on voice, video and data IP, also allowing the exchange of information between operators. CUCM standardized components, by levels, are:

- infrastructure containing the routers, switches and Voice Gateway; the infrastructure level enables exchange of data, voice and video between network devices and applications.

- call control which is responsible for handling calls, controlling device and managing dialing plans.

- an application that is an independent level of the call control, which allows applications to run anywhere on the network, such as Voice Mail, Cisco Unified Meeting Place, Cisco Emergency Responder, as well as of the integration of SOAP, Q.SIG, H.323, MGCP and SIP protocols. The CUCM node in this simulation was located in the carrier operator's website. GNS3 emulator was used to configure the Cisco 3725 router and voice gateway, and Cisco IP Communications application to simulate calls [8].

In the proposed simulation scenario, both locations responsible for each operator simulate the connection to dial the number corresponding to operators, each location having a specific set numbering plan. CUCM was installed on a VMWare virtual machine, with a storage space of $160 \mathrm{G}$ and 8 GRAM. The idea was to simulate an environment as similar as possible to that described in paragraph 2.1., based on the H.323 protocol and SIP
(Session Initiation Protocol) on the VoIP part. SIP, a signalling protocol, found in the application layer of the OSI stack, was used to create, modify, and terminate sessions between two or more participants in the conversation. The H.323 protocol was used between the routers and SIP. Topology was created in GNS3, and CUCM was the connection between the node and the Vmnet8 interface of the virtual machine. Routers were configured to provide connections based on H.323 protocol and SIP to CUCM. Ethernet port configuration and protocol H.323 activation for Voice Gateway are described below:

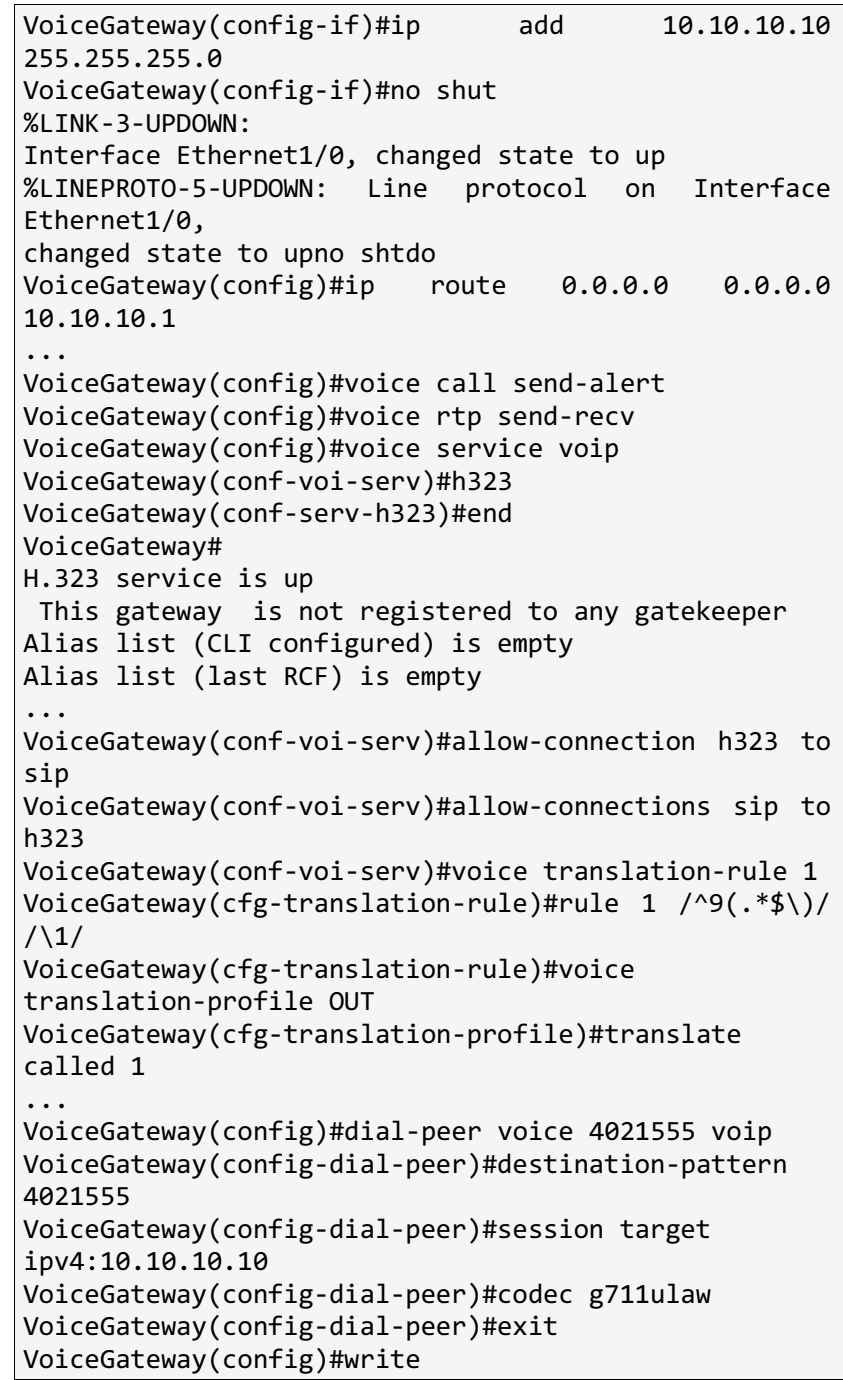

It can be considered that within the carrier network to Voice Gateway, the numbering of the caller and the call recipient may be stored according to SIP. It is possible that, by processing ID and numbering, the interconnection interface between operators can change the identity of the call source. SIP is widely used today as a signalling protocol for VoIP. Use of a PBX (Private Branch Exchange) by the dominant operator, which does not support SIP Trunking, will require a Voice Gateway to connect to the SIP provider. The gateway was placed between the PBX and the SIP provider, using an E1 ISDN (Integrated Services Digital Network) stream via the IP protocol [9]. These settings are not complete and 
are only the initial setup of voice characteristics. H323 calls are activated to SIP and SIP to H323 for Voice Gateway and R1, respectively. Subsequently, the connection for CUCM was configured. Cisco Unified Communications Manager supports several types of Cisco gateways. To configure Voice Gateway, they are used for communication terminals when IP telephony infrastructure communicates with PSTN (Public Switched Telephone Network) or other devices such as PBXs, analogue phones, fax machines, etc. The interfaces specify how the gateway communicates with the PSTN or other devices. The route plan is one of the most important components of a CUCM system. It is an integral part of all call processing operations. Generally, the route plan is the decision maker and instructs the call processing on how to route the calls (Figure 3 ).

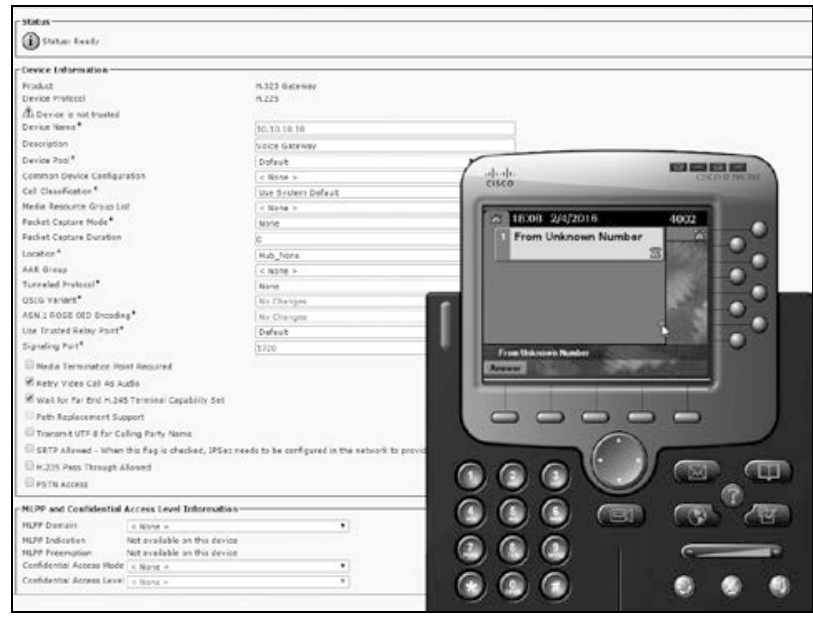

Figure 3. Explanatory scheme on showing an unknown number on the phone display in absence of a routing plan. Sources: Display Capture.

The dial plan describes how calls are processed and routed. This plan can be described based on certain main functions [10], of which the following are listed:

- Endpoint addressing;

- Calling;

- Path selection;

- Digit manipulation, the number of the source can be modified to remove the prefix (Figure 3);

- Call coverage;

- Patterns, the absence of a patterns route, can determine display an unknown number.

\subsection{Testing an interconnection situation}

Since it was not possible to run tests on a mockup with exactly the above-mentioned configuration, similar equipment was used to test those previously described in the paper. In order to attain our goals, we used an environment simulating the infrastructure of some operators, namely the connection between the dominant and the carrier operator. The tests were performed for 3 relevant situations:

Case 1: - one unaltered and one altered port. The call is initiated on a phone with the number $0132130002-$ port 1 (unaltered port), and the recipient mobile phone shows the unaltered number 96850112 , which is also registered in the logs $(\mathrm{ABOIN})$.

The explanatory configuration on the recipient phone screen and the number registration in logs, is described below:

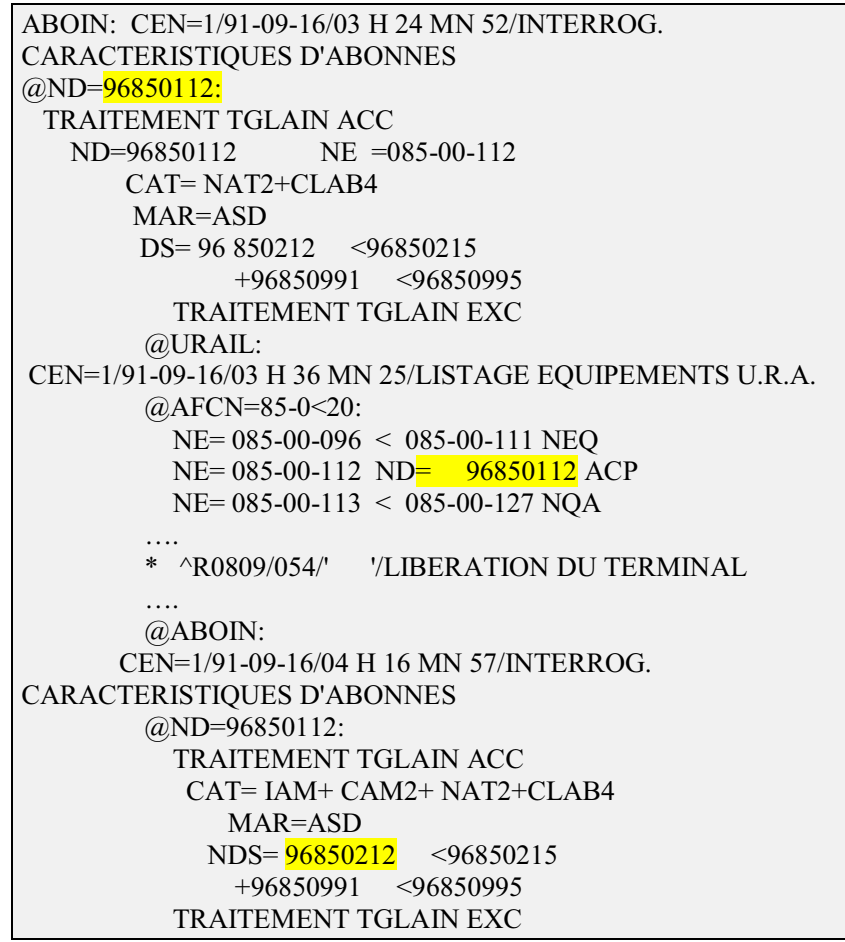

The call is initiated on a phone with the number 0132 140005 - port 2 (altered port), and the recipient mobile phone shows the altered number (the number set in the switch application), i.e. number 96850991.

Case 2: - both ports are altered. The call is initiated on a phone with the number 0132130002 - port 1 (altered port), and the recipient mobile phone shows the altered number 96850991 (the number set in the switch application) which is registered, and thus featured in the logs (ABOIN). The call is initiated on a phone with the number 0132140005 - on port 2 (altered port), and the recipient mobile phone shows the altered number (the number set in the switch application), i.e. the number 96 850 991, which was also noticed in the logs (ABOIN)

Case 3: - both ports altered. The call is initiated on a phone with the number 0132130002 - port 1 (altered), and the recipient mobile phone shows the altered number 96850991 (the number set in the switch application) which was also noticed in the logs (ABOIN) The call is initiated on a phone with the number 0132140005 - port 2 (altered), and the recipient mobile phone shows the altered number (the number set in the switch application), i.e. the number 96850995 , which was also seen in the $\operatorname{logs}(\mathrm{ABOIN})$. 


\section{Traffic and voice transmission quality analysis}

\subsection{The carrier operator and/or service provider has routed into the dominant operator's network international traffic and/or national traffic originated in other networks than its own}

Following the ANCOM decision on the standardization of national and international rates (2007), the carrier operator and/or service provider has reduced traffic (starting in the $36^{\text {th }}$ month, Figure 4), but only for a short time period.

a) One argument for the major increase in traffic compared to the amount established in the agreement $(30,000$ minutes/month) is given by the small number of subscribers declared by the carrier operator and/or service provider in relation to the dominant operator.

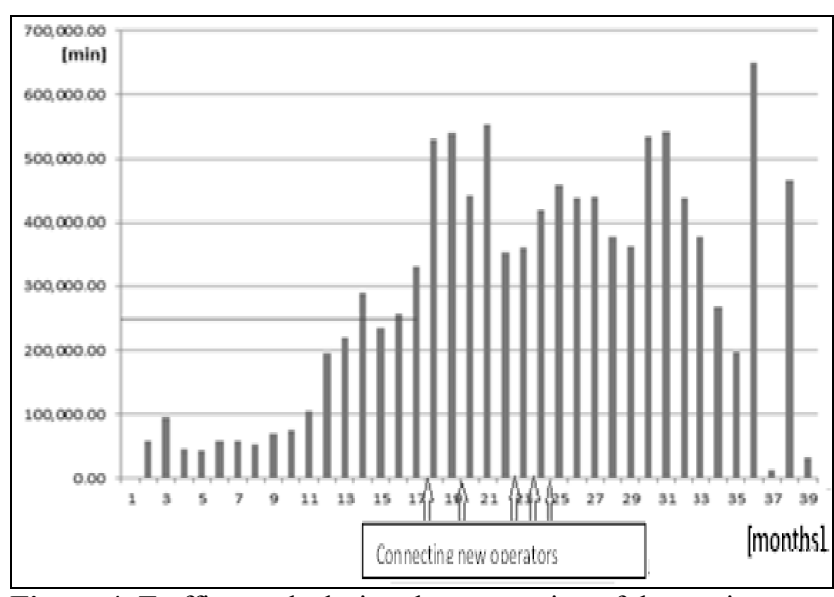

Figure 4. Traffic trends during the connection of the carrier operator and/or service provider with the dominant operator

Thus, during traffic monitoring by the dominant operator the given traffic values (Figure 5) are real, i.e. in accordance with the number of subscribers declared by the carrier operator and/or service provider.

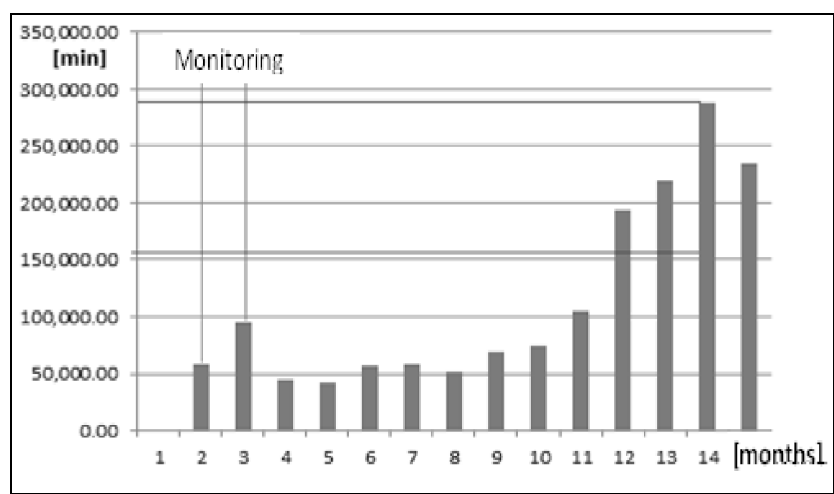

Figure 5. Traffic trends during monitoring by the dominant operator.

b) During the period under review, according to the existing agreement between the carrier operator and/or service provider and another dominant operator on the market, prepaid cards and smart calls are also used by subscribers of those networks. The service operator, offering conditions for routing traffic for subscribers of the other dominant operator and international traffic to subscribers of the dominant operator. The carrier operator and/or service provider would use card count-type cards by which the subscriber, dialing a phone number, after entering the account number, could access any other number in the telecommunication networks connected to the card issuer (card issuer identifier code), these short numbers being called carrier selection code. By using the smart call service, a subscriber can dial the desired number after previously entering a "short" phone number. The caller's ID may be replaced with another, pertaining to the carrier operator and/or service provider network, so that when reaching destination, the caller may be considered to be the carrier operator and/or service provider's own subscriber.

c) Use of traffic routing through VoIP protocol (allows changing caller identity upon entering another network), by the carrier operator and/or service provider, illustrates the fact that the carrier operator and/or service provider will be able to route national and international traffic to the dominant operator's network.

Thus, after converting voice signals and preparing the packets, upon entry into the operator's network operator and/or service provider, the true identity of the caller is replaced with an ID (caller number) pertaining to the transit network (of the carrier operator and/or service provider), and upon destination/reception the call appears as coming from the transit network and not the real one. Although ITU-T recommendations require just one signalling change for establishing communications in a national area, it can be noted that two changes are made in the signalling system, namely, into and out of the carrier operator and/or service provider network.

d) During the period under review, the carrier operator and/or service provider concluded a large number of interconnection agreements with various companies providing telecommunication services, concentrating and routing their traffic to subscribers of the dominant operator network. Routing other operators' traffic to the dominant operator network is also apparent from Figure 5 , where traffic increases are highlighted every time a new network connects to the carrier operator and/or service provider network [4].

e) In addition to the amount of incoming traffic (Figure $6 a)$, the dominant operator also monitored the average duration of incoming calls (Figure 6b) tolerating the behavior of the carrier operator and/or service provider.

Thus, there is a sudden increase in the average duration of calls from 1.5 minutes in the first 20 days to more than 4 minutes in the next 5 days, which indicates that the carrier operator and/or service provider began to route additional traffic (possibly international traffic) from the one originated in the home network to the dominant operator network. 

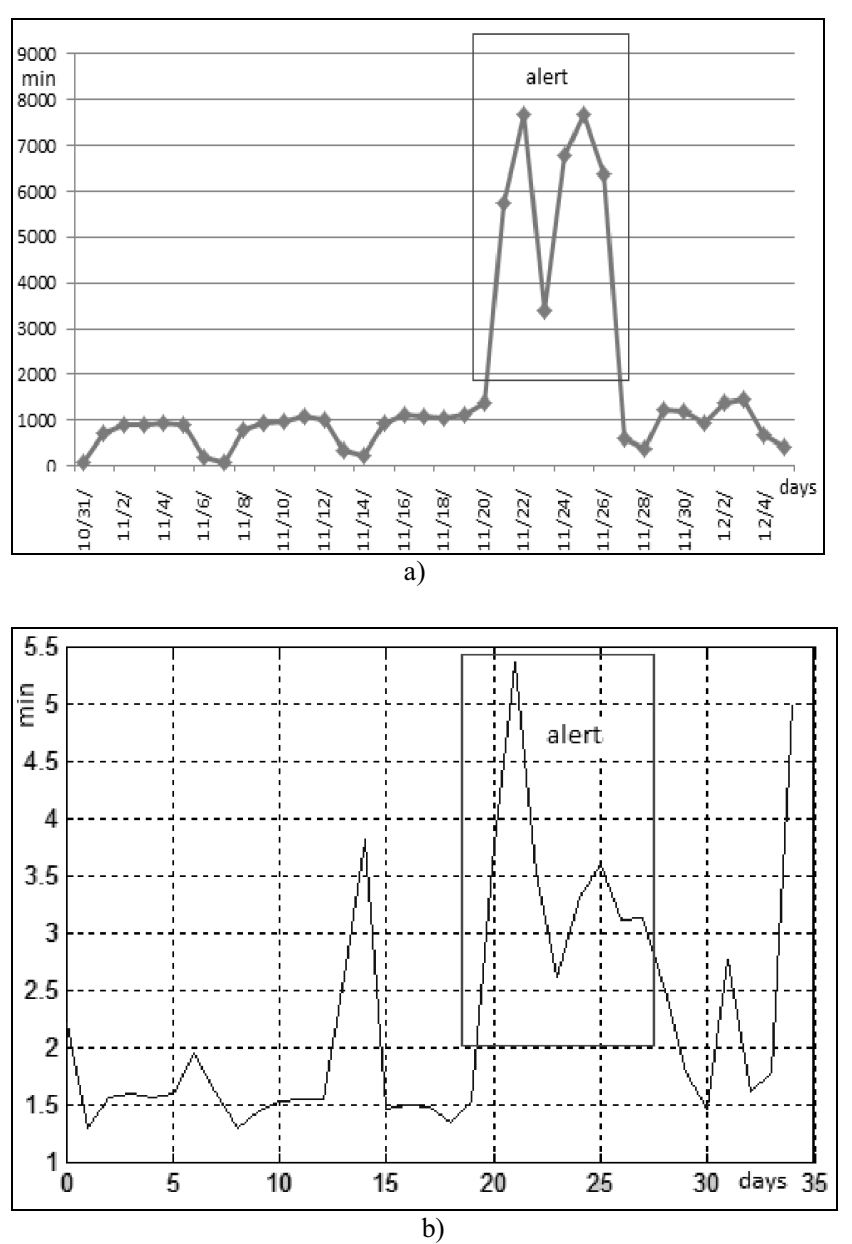

Figure 6. Explanatory scheme on incoming traffic (a) and average duration of calls (b).

\subsection{The quality of VolP communications, the relationship between the carrier operator and/or service provider and the dominant operator}

a) Quality parameters established for national and international communications, valid for both the carrier operator and/or service provider and the dominant operator, are [11]:

- the link upload domain, necessary to enable the carrier operator and/or service provider to use VoIP [12], with values ranging between 0.2 - 0.4 Erlang [13]; exceeding the upper threshold involves increasing waiting "queue" and thus loss of packets;

- the route loading indicator, ranging between $10-90 \%$ Erlang B, determines the probability for a call to be blocked; very high values will highlight the poor quality of equipment and software used by the carrier operator and/or service provider; reaching the upper limit cannot be accepted even in the case of route transport capacity falling below $20 \%$;

- the percentage of erroneous seconds $<1.4 \%$ (14 missed calls in 1,000); reaching the upper limit is not acceptable for national/international traffic;

- Answer Seizure Ratio (ASR)> 50\%, being dependent on the subscriber's presence in the network.

The above values, listed in the studied VoIP communications, do not meet the quality requirements desired by subscribers and do not fall into the general characteristics of voice communications, namely, ID change, interruptions and lengthy shutdowns.

b) The above-mentioned parameters as quality indicators are insufficient to ensure that the traffic routed through and originated on the carrier operator and/or service provider network maintains its quality levels upon arrival to the dominant network. VoIP-based service quality was contested by many international operators. Quality problems have only reached acceptable levels in 2008, by implementing Internet Protocol version 8 (IPv8), which allowed controlling packets carrying voice information and reducing the number of lost packets. Full implementation of this version, by all users, was due to be completed in 2013, which never happened.

c) As the transmission of voice packets can be affected by lack of synchronization occurring between the telecommunication network and telecommunication terminal adapters, resulting in loss of packets, VoIP communications are regarded as having a "best effort" quality level. However, if the bandwidth is much larger than that of an E1 stream ( $>2 \mathrm{Mbps})$, then communications are not affected by packet loss, and transmission performance is "premium rate".

d) The existence of two changes in the signalling system drastically affects the quality of communications. In this respect, reference is made to the poor quality of communications before 1989, in Romania, when analogue communication systems were used, requesting two changes in the signalling system (Rotary [14] and Pentaconta technologies).

\section{Conclusions}

Performing simulations is required for the numerical analysis of the conflict situation arising between telecommunication operators. Thus, it has been observed that there is a possibility that numbers in a VoIP network be converted into another numbering plan based on preestablished settings. If the call source number does not correspond to a specific predefined pattern, the dominant operator may convert that number into one of its own network. Using a prefix to access the dominant operator network can result in conversion of the call source number to match the numbers in that network by removing the prefix and changing it. In the case presented in our paper, during the period under review, the carrier operator and/or service provider routed both traffic originated in other national networks than its own and international traffic to the dominant operator network, and the dominant operator allowed the routing of this traffic voluntarily and knowingly. The quality of VoIP communications, in the connection between the dominant operator and the carrier operator and/or service provider, did not meet the quality parameters required for national and international communications. The quality standard for this type of communication was achieved only after the implementation of Internet Protocol version 8, started in 2009 and expected to be completed in 2013, although currently in Romania there are communication networks using old versions of the Internet Protocol, namely IPv6. 


\section{References}

1. M. Popescu, Telecommunications (in Romanian) Ed. Universitaria, Craiova (2008)

2. ANS TransLocal for optimal flexibility, Ericsson Diax A/S (2010)

http://archive.ericsson.net/service/internet/picov/get? DocNo=1/28701-FGC101591\&L.

3. M.M. Eissa, M. M. Elmesalawy, M.M.A. Hadhoud, Wide Area Monitoring System based on the third generation Universal Mobile Telecommunication System for event identification, International Journal of Electrical Power \& Energy Systems, 69, pp 34-47 (2015)

4. V. Croitoru, D. Geleriu, The report expertise in telecommunications specialty (not published), Bucharest (2013).

5. J. Snader, VPNs Illustrated: Tunnels, VPNS and IPsec, Ed. Addison Wesley Professional (2005)

6. GNS3 software, https://www.gns3.com/

7. Cisco Unified Communications Manager http://www.cisco.com/c/en/us/products/unifiedcommunications/unified-communications-managercallmanager/index.html
8. Cisco IP Communicator http://www.cisco.com/c/en/us/products/collaboration -endpoints/ip-communicator/index.html

9. S. Brent, CCNA Voice Lab Manual, Cisco Press, Indianapolis, Indiana (2013)

10. J. Cioara, V. Michael, CCNA Voice 640-461 Official Cert Guide, Cisco Press, Indianapolis, Indiana (2011)

11. T. Vaughan, Multimedia: Making It Work, $8^{\text {th }}$ Edition, Ed. McGraw-Hill (2011)

12. P. Carden, Building Voice over IP, Network computing (2000)

http://www.networkcomputing.com/netdesign/1109v oipfull.html\#two

13. M.C. Popescu, N. Mastorakis, New Aspect on Wireless Communication Networks, International Journal of Communications, Issue 1, 3, pp.34-43, (2009) http://www.naun.org/journals/communications/

14. A. Calvo, Compañía Telefónica Nacional de España: From subsidiary to leader, in Telecommunications Conference (HISTELCON), Second IEEE Region 8 Conference on the History of, pp.1-7, Madrid (2010) 